\title{
Classification of Lactobacillus plantarum by Restriction Endonuclease Analysis of Total Chromosomal DNA Using Conventional Agarose Gel Electrophoresis
}

\author{
M.-L. JOHANSSON, M. QUEDNAU, S. AHRNÉ, AND G. MOLIN* \\ Laboratory of Food Hygiene, Division of Food Technology, Lund University, Lund, Sweden
}

\begin{abstract}
A total of 17 Lactobacillus plantarum strains that originated from different environments and 24 reference strains were classified by performing a restriction endonuclease analysis of total chromosomal DNAs digested with $E c o$ RI, HindIII, and ClaI, and the resulting patterns were visualized after the fragments were separated according to size by agarose gel electrophoresis. The patterns were analyzed by using the Pearson product moment correlation coefficient and the unweighted pair group algorithm with arithmetic averages. All but two L. plantarum strains formed a cluster that was separated from the reference strains at a similarity level of $29 \%$ (cluster 1). The two remaining L. plantarum strains (cluster 2) merged with cluster 1 at a level of similarity of $\mathbf{2 8 \%}$. The reference strains formed four additional clusters, and four reference strains were stragglers. Cluster 3 (three Lactobacillus pentosus strains) and cluster 4 (including Pediococcus acidilacti CCUG 32235 ${ }^{\mathrm{T}}$ [T $=$ type strain] and Lactobacillus fermentum ATCC $14931^{\mathrm{T}}$ ) merged with cluster 1 at a level of similarity of $25 \%$. Cluster 5 comprised 10 Lactobacillus reuteri strains, and cluster 6 contained the type strains of Lactobacillus amylovorus, Lactobacillus gasseri, and Lactobacillus vaginalis. Cluster 1 (L. plantarum) was divided into three subclusters, and this subdivision reflected to some extent certain phenotypic features of presumed ecological significance, including the ability to adhere to intestinal mucosa (subcluster 1c) and starch and glycogen degradation (subcluster 1a). A principal-component analysis significantly distinguished the strains belonging to different species. Also, the subcluster 1c strains could be separated from the rest of the $L$. plantarum strains. The results of restriction endonuclease analysis of total chromosomal DNA were found to be reproducible, and this method can be used to (i) differentiate between similar strains belonging to the same Lactobacillus species and group strains within a species, (ii) distinguish between strains of different Lactobacillus species, and (iii) place strains in specific Lactobacillus species.
\end{abstract}

The genus Lactobacillus is heterogeneous, and the relationships among the different species have been studied by reverse transcriptase sequencing of 16S rRNAs (2). Larger evolutionary distances between species have been revealed clearly, but the relationships among more closely related species are muddled. Furthermore, rRNA gene sequencing is useless for classification below the species level; for example, the 16S rRNA gene sequences of Lactobacillus plantarum and Lactobacillus pentosus are identical, and the same is true for the sequences of Lactobacillus casei and Lactobacillus rhamnosus $(2,12)$.

Workers have developed a method in which the genomes of Lactobacillus strains are characterized by performing a restriction endonuclease analysis (REA) of chromosomal DNAs and then visualizing the resulting fragment patterns after agarose gel electrophoresis is performed (14). This method has been used to classify Lactobacillus reuteri strains (13). The general advantage of REA is that it provides a unique genomic fingerprint of the whole chromosomal DNA for each strain. The presence of multiple band patterns, which are obtained by using restriction endonucleases that are able to split the DNA at many sites, means that a taxonomic analysis can be based on a large number of genomic features (DNA fragment sizes) for each strain. At the strain level, the resolution of REA for $L$. plantarum is better than the resolution of ribotyping (6) or randomly amplified polymorphic DNA analysis (7). Furthermore, it has been suggested that this method can identify strains to the species level (13). Thus, REA might supplement

${ }^{*}$ Corresponding author. Mailing address: Division of Food Technology, Chemical Center, P.O. Box 124, S-221 00 Lund, Sweden.
16S rRNA gene sequencing by providing data for the lower taxonomic levels.

$L$. plantarum is frequently used as a starter culture in lactic acid-fermented foods (9) and has also been isolated from human intestinal mucosa (10). The only previous major taxonomic study of $L$. plantarum at the interspecies level was performed by Dellaglio et al. (3), who divided strains identified phenotypically into two DNA-DNA homology groups. One of these groups was later designated L. pentosus (16).

The aims of this study were to use an improved and technically somewhat simplified REA method to classify $L$. plantarum strains isolated from the mucosa of human intestines and from lactic acid-fermented foods and to compare these strains with strains belonging to other species, including the $L$. pentosus type strain and a set of $L$. reuteri strains.

\section{MATERIALS AND METHODS}

Strains and culture conditions. The strains which we used are listed in Table 1. All of the $L$. plantarum, $L$. pentosus, and $L$. reuteri strains had previously been genetically identified to the species level by performing DNA-DNA hybridization experiments with the type strains or by ribotyping $(6,13)$. The strains were grown and stored as described previously (13).

Preparation of DNA and restriction endonuclease digestion. Chromosomal DNAs were prepared after the strains were grown overnight in stationary cultures at $37^{\circ} \mathrm{C}$ in $100-\mathrm{ml}$ portions of Lactobacillus carrying medium (4) containing $1 \%$ glucose. Cells were harvested by centrifugation, washed once in $10 \mathrm{ml}$ of TES (50 mM NaCl, $100 \mathrm{mM}$ Tris, $70 \mathrm{mM}$ disodium EDTA; $\mathrm{pH} 8.0$ ), and suspended in $3 \mathrm{ml}$ of TES supplemented with $25 \%$ (wt/vol) sucrose. Then lysozyme $(120 \mathrm{mg}$; grade VI; Sigma Chemical Co., St. Louis, Mo.) and $140 \mathrm{U}$ of mutanolysin (Sigma) were added. The cells were incubated at $42^{\circ} \mathrm{C}$ for $2 \mathrm{~h}, 3 \mathrm{mg}$ of proteinase $\mathrm{K}$ (type XI; Sigma) was added, and the preparation was incubated at $37^{\circ} \mathrm{C}$ for 30 min. After cooling, $1 \mathrm{ml}$ of a $5 \% \mathrm{NaCl}$ solution, $1 \mathrm{ml}$ of $20 \%$ sodium dodecyl sulfate, and $11.4 \mathrm{ml}$ of TES were added to the cell suspension. If necessary, the suspension was gently stirred with a glass rod until it became homogeneous, and then it was heated at $65^{\circ} \mathrm{C}$ for $15 \mathrm{~min}$. The cell lysate was extracted once with 
TABLE 1. Strains used in this study and their sources of isolation

\begin{tabular}{|c|c|c|}
\hline Strain $^{a}$ & Source of isolation & $\begin{array}{c}\text { Strain } \\
\text { no." }\end{array}$ \\
\hline Lactobacillus plantarum ATCC $14917^{\mathrm{T}}$ & Pickled cabbage & 1 \\
\hline Lactobacillus plantarum $386^{\text {c.d }}$ & Human rectum & 2 \\
\hline Lactobacillus plantarum $299^{\text {cid }}$ & Human colon & 3 \\
\hline Lactobacillus plantarum $105^{c, d}$ & Human transversum & 4 \\
\hline Lactobacillus plantarum $275^{\text {c.d }}$ & Human colon & 5 \\
\hline Lactobacillus plantarum $299 \mathrm{v}^{c i c^{\circ}}$ & $\begin{array}{l}\text { Sour dough, human } \\
\text { intestine }\end{array}$ & 6 \\
\hline Lactobacillus plantanum So5 $f$ & & 7 \\
\hline Lactobacillus plantarum $256^{c \cdot g}$ & Silage & 8 \\
\hline Lactobacillus plantanum $36 \mathrm{E}^{c / t}$ & Silage & 9 \\
\hline Lactobacillus plantarum $79^{c i}$ & Nigerian pito & 10 \\
\hline Lactobacillus plantarum $107^{\circ i}$ & Nigerian ogi & 11 \\
\hline Lactobacillus plantarum $98^{c, i}$ & Nigerian ogi & 12 \\
\hline Lactobacillus plantarum $95^{c, i}$ & Nigerian ogi & 13 \\
\hline Lactobacillus plantarum $53^{c, i}$ & Nigerian ogi & 14 \\
\hline Lactobacillus plantarum $97^{c, i}$ & Nigerian ogi & 15 \\
\hline Lactobacillus plantarum $101^{c i}$ & Nigerian ogi & 16 \\
\hline Lactobacillus plantarum $120^{c i}$ & Nigerian ogi & 17 \\
\hline Lactobacillus plantarum $44^{c i}$ & Nigerian ogi & 18 \\
\hline Lactobacillus plantarum ATCC 8014 & Corn silage & 19 \\
\hline "Lactobacillus plantarum" $\mathrm{CH}^{i, k}$ & & 20 \\
\hline "Lactobacillus plantarum" $442^{\prime . l}$ & $\begin{array}{l}\text { Fermented } \\
\text { cucumber }\end{array}$ & 21 \\
\hline Lactobacillus pentosus ATCC $8041^{\mathrm{T}}$ & Corn silage & 22 \\
\hline Lactobacillus reuteri DSM $20016^{\mathrm{T}}$ & & 23 \\
\hline Lactobacillus reuteri DSM $20015^{m}$ & & 24 \\
\hline Lactobacillus reuteri $\mathrm{N} 2 \mathrm{LC}^{n}$ & Rat intestine & 25 \\
\hline Lactobacillus reuteri N5IC: $2^{m}$ & Rat intestine & 26 \\
\hline Lactobacillus reuteri $\mathrm{N}_{2} \mathrm{~J}^{m}$ & Rat intestine & 27 \\
\hline Lactobacillus reuteri $\mathrm{N} 5 \mathrm{D}: 1^{\prime \prime}$ & Rat intestine & 28 \\
\hline Lactobacillus reuteri $1044^{\prime \prime}$ & Porcine intestine & 29 \\
\hline Lactobacillus reuteri $8557: 1^{d, m}$ & Human & 30 \\
\hline Lactobacillus reuteri $1048^{\prime \prime}$ & Porcine intestine & 31 \\
\hline Lactobacillus reuteri $8557: 3^{d, m}$ & Human & 32 \\
\hline
\end{tabular}

Lactobacillus fermentum ATCC $14931^{\top}$

Pediococcus acidilacti CCUG $32235^{\top}$

Lactobacillus gasseri DSM $20243^{\mathrm{T}}$

Lactobacillus vaginalis CCUG $31452^{\mathrm{T}}$

Lactobacillus amylovorus DSM $20532^{\mathrm{T}}$

Lactobacillus helveticus DSM $20075^{\mathrm{T}}$

"Lactobacillus plantarum" ATCC 10776 "

Erysipelothrix rhusiopathiae ${ }^{a}$ CCUG $221^{\mathrm{T}}$

Pediococcus pentosaceus CCUG $32205^{\mathrm{T}}$

"ATCC, American Type Culture Collection, Rockville, Md.; DSM, Deutsche Sammlung von Mikroorganismen und Zellkulturen, Braunschweig, Germany; CCUG, Culture Collection of the University of Gothenburg, Gothenburg, Sweden.

${ }^{b}$ Strain numbers used in Fig. 3 and 4.

c Genetically identified by DNA-DNA hybridization and affiliated with $L$. plantarum (6)

${ }^{d}$ Isolated by Molin et al. (10).

e Described by Johansson et al. (5)

Starter culture obtained from the Swedish Sugar Company, Arlöv, Sweden.

${ }^{g}$ Sven Lindgren, Department of Microbiology, Uppsala University, Uppsala, Sweden.

${ }^{h}$ Siv Ahrné, Laboratory of Food Hygiene, Department of Food Technology, Lund University, Lund, Sweden.

${ }^{i}$ Isolated by Johansson et al. (8)

${ }^{j}$ Genetically identified by DNA-DNA hybridization and affiliated with $L$. pentosus (6).

${ }^{k}$ Starter culture obtained from Christian Hansen Laboratories, Copenhagen, Denmark.

${ }^{l}$ H. Fleming, University of North Carolina, Raleigh.

${ }^{m}$ Genetically identified by DNA-DNA hybridization as $L$. reuteri and studied by REA (13).

${ }^{n}$ Genetically identified by DNA-DNA hybridization and found to belong to neither $L$. plantarum nor L. pentosus (6).

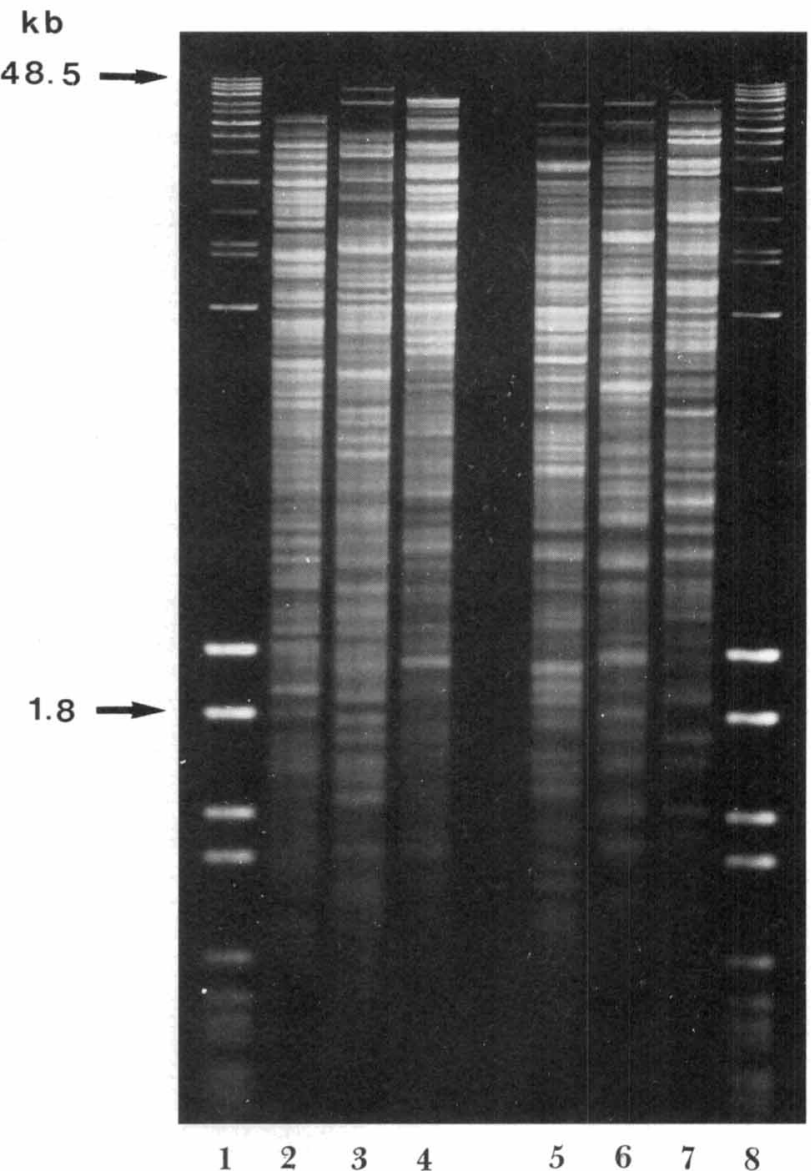

FIG. 1. Agarose gel electrophoresis of L. plantarum ATCC 8014 (lanes 2 through 4) and "L. plantanim" ATCC 10776 (lanes 5 through 7) DNAs cleaved with HindIII (lanes 2 and 5), ClaI (lanes 3 and 6), and EcoRI (lanes 4 and 7). Lanes 1 and 8 contained a high-molecular-weight DNA marker (Bethesda Research Laboratories) and DNA molecular weight marker VI (Boehringer Mannheim). The bands between the arrows were compared.

redistilled phenol-chloroform (1:1) and once with chloroform-isoamyl alcohol (24:1). The DNA was then precipitated with ethanol at $-20{ }^{\circ} \mathrm{C}$.

The chromosomal DNA was separated from covalently closed circular plasmid DNA (most of the plasmid DNA) by dye buoyant density centrifugation in a $\mathrm{CsCl}$ gradient containing ethidium bromide (1). Some linear and open circular forms of plasmid DNA were still present in the preparation, but they contributed very little to the final results (13) (data not shown). The concentration of chromosomal DNA was monitored with a fluorometer (model TKO 100; Hoefer Scientific Instruments, San Francisco, Calif.).

DNA $(0.75 \mu \mathrm{g})$ was separately digested at $37^{\circ} \mathrm{C}$ for $4 \mathrm{~h}$ with $10 \mathrm{U}$ of HindIII, ClaI, or EcoRI (Boehringer Mannheim). Ståhl et al. (14) and Stăhl and Molin (13) used Asp 718 instead of HindIII, but digestion with Asp 718 resulted in too many large bands to be optimal for the method which we used.

Electrophoresis and scanning of gels. Gel electrophoresis was carried out by using submerged horizontal $0.9 \%$ agarose (ultrapure DNA grade; low electroendoosmosis; Bio-Rad Laboratories, Richmond, Calif.) slab gels ( 150 by 235 $\mathrm{mm})$. A high-molecular-weight DNA marker $(0.2 \mu \mathrm{g})$ containing DNA fragments having molecular sizes ranging from 8.5 to $48.5 \mathrm{~kb}$ (Bethesda Research Laboratories) and $0.5 \mu \mathrm{g}$ of DNA molecular weight marker VI containing DNA fragments having molecular sizes ranging from 0.2 to $2.2 \mathrm{~kb}$ (Boehringer Mannheim) were used as standards. In addition, pHC 79 digested with EcoRI (molecular size, $6.4 \mathrm{~kb}$; Boehringer Mannheim) was added to each well as an internal standard. Minimal band distortion and maximal sharpness were achieved by applying the sample DNA in Ficoll loading buffer ( $2 \mathrm{~g}$ of Ficoll, $8 \mathrm{ml}$ of water, $0.25 \%$ bromphenol).

Gels were electrophored at a constant voltage of $40 \mathrm{~V}$ for $18 \mathrm{~h}$ at $8.5^{\circ} \mathrm{C}$; the buffer ( $89 \mathrm{mM}$ Tris, $23 \mathrm{mM} \mathrm{H} \mathrm{PO}_{4} .2 \mathrm{mM}$ sodium EDTA; $\mathrm{pH} 8.3$ ) was recirculated during electrophoresis. Then the gels were stained for $20 \mathrm{~min}$ in ethidium bromide $(2 \mu \mathrm{g} / \mathrm{ml})$, destained in distilled water, visualized at $302 \mathrm{~nm}$ with a UV 


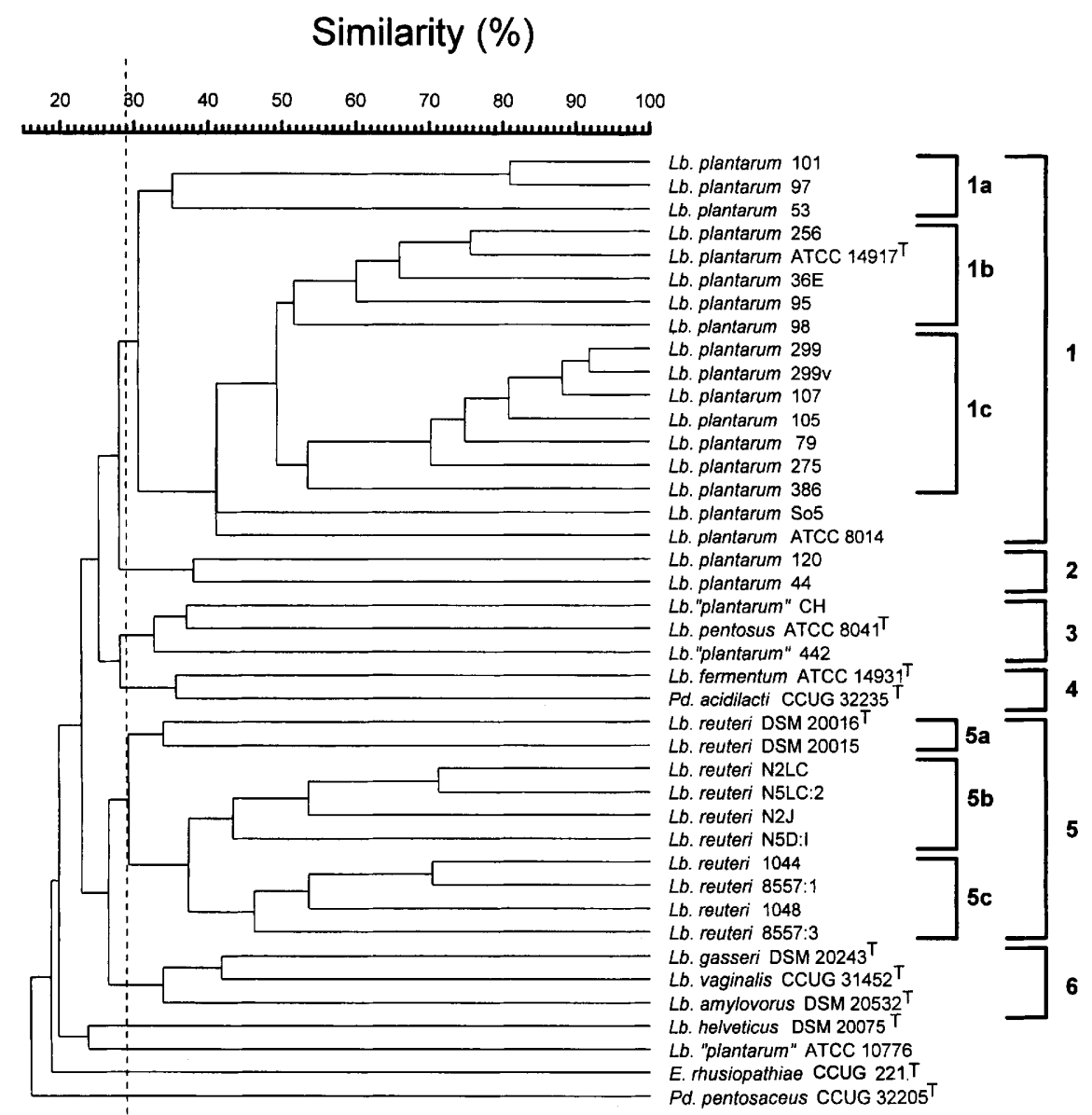

FIG. 2. Dendrogram of the strains tested based on Pearson product moment correlation coefficients and UPGMA clustering. Lb., Lactobacillus; Pd., Pediococcus; E., Erysipelothrix.

transilluminator (UVF, Inc., San Gabriel, Calif.), and photographed. This method of performing gel electrophoresis gave well-distributed and relatively well-separated bands down to a molecular size of about $1.8 \mathrm{~kb}$ (Fig. 1)

Reading band patterns. Band patterns on photographic negatives were scanned with a laser densitometer (UltroScan XL; LKB-Produkter AB, Bromma, Sweden). The level of resolution was 1,000 points per lane, and each lane was about $50 \mathrm{~mm}$ long. Data were obtained by using the LKB 2400 GelScan XL software package (LKB-Produkter $\mathrm{AB}$ ). The band patterns were normalized, and background noise was subtracted by using the rolling-disc algorithm of the GelCompar 3.0 program (Applied Maths, Kortrijk, Belgium). The data from the three experiments (the experiments performed with HindIII, ClaI, and EcoRI) were combined for each strain by using GelCompar 3.0.

Mathematical analyses. The data set was analyzed by using the Pearson product moment correlation coefficient and the unweighted pair group algorithm with arithmetic averages (UPGMA) (11). The Pearson correlation coefficients and the UPGMA values were calculated for the complete data set, and the GelCompar 3.0 program was used to do this. Our analysis also involved a principal-component analysis (PCA) performed with the strains affiliated with $L$. plantarum, $L$. reuteri, and $L$. pentosus, as well as the $L$. plantarum strains alone (using GelCompar 3.0).

Reproducibility. DNAs from the following strains were analyzed in duplicate (the chromosomal DNA of each strain was prepared twice, and the samples were electrophoresed on different gels): L. plantanum 53,97 , ATCC $14917^{\mathrm{T}}$ (T = type strain), 36E, ATCC $8014,299,105,275$, and 120 . This analysis was followed by a cluster analysis.

Phenotypic analysis. All $L$. plantarum strains and $L$. pentosus ATCC $8041^{\mathbf{T}}$ were analyzed by using API $50 \mathrm{CH}$ kits (API System, Montalieu, Vercieu, France) as described previously (10) at both 30 and $37^{\circ} \mathrm{C}$. The profiles obtained at the two temperatures were combined for each strain (in all, 98 characters were examined) and were compared by using the Jaccard coefficient followed by UPGMA clustering.

\section{RESULTS}

Reproducibility. The selected strains analyzed in duplicate merged in the cluster analysis, with no strains in between. This indicated that most of the strains in this study could be distinguished by the REA method which we used. In almost all cases the levels of similarity obtained for duplicate runs of the same strain were in the range from of 90 to $95 \%$.

Cluster analysis. The cluster analysis in which we used the Pearson product moment correlation coefficient followed by UPGMA resulted in the dendrogram shown in Fig. 2. Five clusters were defined at a similarity level of $29 \%$. Cluster $1(L$. plantarum) comprised $17 \mathrm{~L}$. plantarum strains that originated from both human intestines and fermented foods and feeds, including $L$. plantarum ATCC $14917^{\mathrm{T}}$ and ATCC 8014 . Three distinct subclusters were also identified, and these subclusters merged at similarity levels of 54,52 , and $35 \%$ (Fig. 2). $L$. plantarum So5 and ATCC 8014 appeared to be stragglers within cluster 1 , merging with the other strains at a similarity level of $42 \%$.

Cluster 2 comprised two L. plantarum strains. These strains merged with cluster 1 at a similarity level of $28 \%$ (Fig. 2).

Cluster 3 (L. pentosus) contained one isolate from pickled cucumber, one commercial starter culture, and $L$. pentosus ATCC $8041^{\mathrm{T}}$ (Fig. 2). 


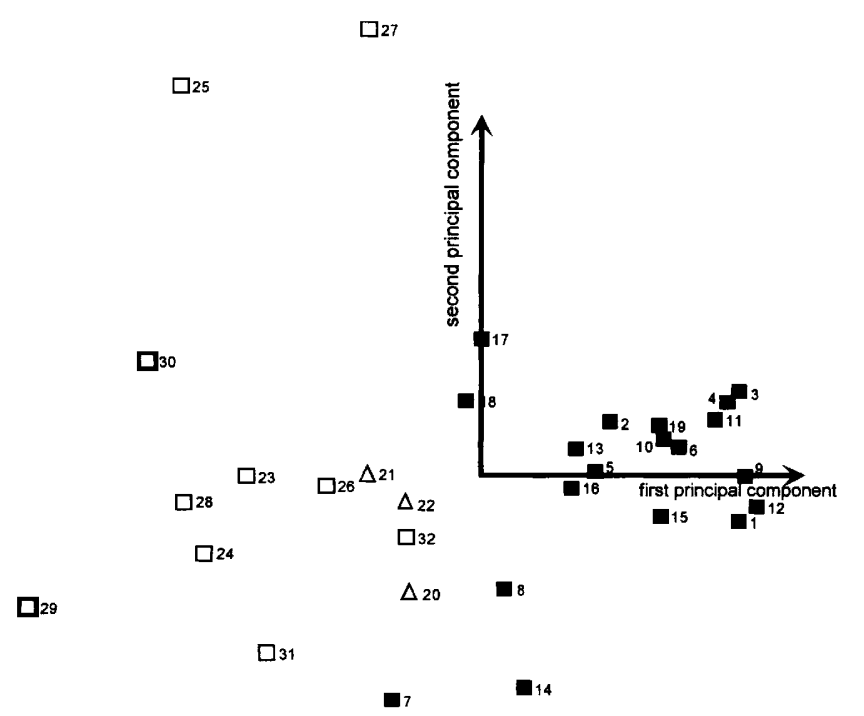

FIG. 3. First- and second-principal-component scores for L. plantarum (ם), $L$. reuteri $(\square)$, and $L$. pentosus $(\triangle)$ strains. The projection was based on the complete data set. The numbers are strain numbers (Table 1 ).

Cluster 4 included two type strains, Pediococcus acidilacti CCUG 32235 and Lactobacillus fermentum ATCC 14931 (Fig. 2).

Cluster 5 (L. reuteri) comprised 10 L. reuteri strains, including $L$. reuteri DSM $20016^{\mathrm{T}}$ and DSM 20015. Cluster 5 could be divided into three subclusters, subclusters 5 a through $5 \mathrm{c}$, which merged at similarity levels of 46,43 , and $34 \%$, respectively (Fig. 2). Subcluster 5b contained strains isolated from rat intestines, and subcluster $5 \mathrm{c}$ included strains that originated from humans and pigs (Fig. 2). L. reuteri DSM 20016 ${ }^{T}$ and DSM 20015 (subcluster 5a) merged with cluster 5, but at a lower level of similarity (29\%).

A sixth cluster, cluster 6, contained three type strains, Lactobacillus gasseri DSM 20243, Lactobacillus vaginalis CCUG 31452, and Lactobacillus amylovorus DSM 20532 (Fig. 2).

Three type strains and one reference strain formed onemember clusters (Fig. 2).

PCA. The PCA and accompanying statistical evaluation of $L$. plantarum, $L$. pentosus, and $L$. reuteri strains revealed that there were three groups (coinciding with the species affiliations) that were significantly separated from each other (Fig. 3) (all data are not shown). Strains that were located peripherally in each group were removed and tested individually to determine their group affiliations. This analysis showed that each strain was more closely related to its assigned species than to any of the other species (data not shown). The L. plantarum group of strains (clusters 1 and 2) was more compact than the L. reuteri group of strains (Fig. 3) (data not shown).

A separate PCA was performed with the $L$. plantarum strains, and the strains in subcluster 1c formed a compact group that was separated from most of the other strains by both the first and second principal components (Fig. 4). It is also noteworthy that the strains in subcluster $1 b$ (Fig. 2) are the strains that merged closest to cluster $1 \mathrm{c}$ in the PCA. According to the PCA plot, L. plantarum So5 and 53 are the strains that are most distantly related to subcluster $1 \mathrm{c}$ according to the first principal component, while $L$. plantarum 44 and 120 are the strains that are most distantly related according to the second principal component.

Phenotype. We compared the abilities of the different $L$.

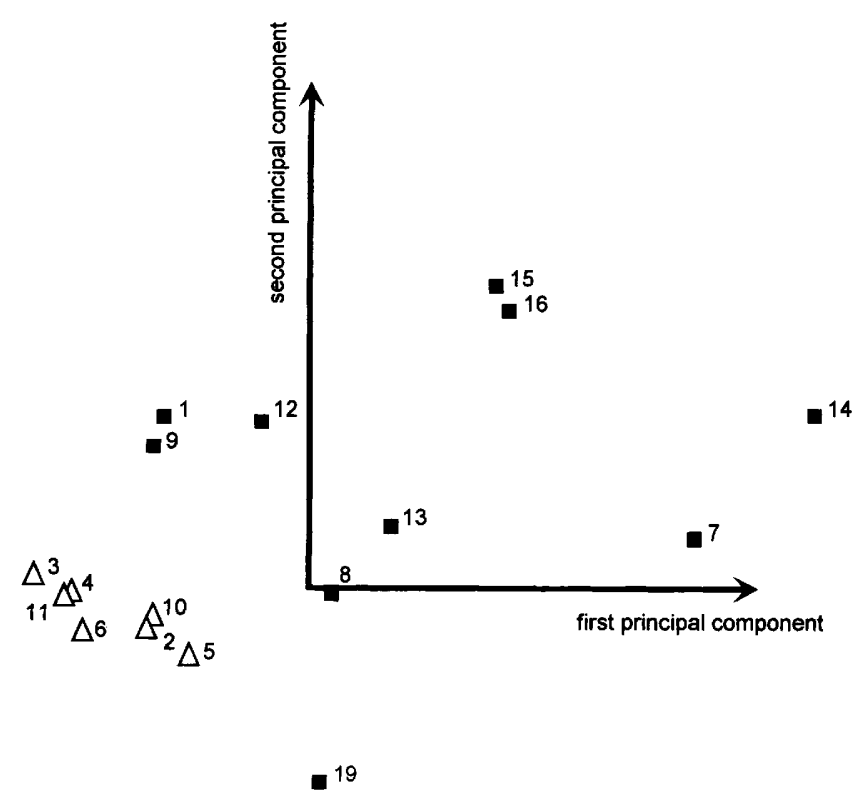

- 18

17

FIG. 4. First- and second-principal-component scores for $L$. plantarum strains, based on the complete data set. Symbols: $\Delta, L$. plantarum strains belonging to subcluster $1 \mathrm{c} ; \mathbf{\square}$, other $L$. plantarum strains. The numbers are strain numbers (Table 1).

plantarum strains (and L. pentosus ATCC $8041^{\mathrm{T}}$ ) to ferment different carbohydrates (as determined by API $50 \mathrm{CH}$ tests), and the resulting dendrogram is shown in Fig. 5. The clustering in this dendrogram resembles in several respects the clustering obtained with the REA. However, strain ATCC 10776 was misidentified by API $50 \mathrm{CH}$ tests as a L. plantarum strain, and the phenotypic tests failed to group the strains in subcluster $1 \mathrm{c}$ in accordance with their genomic relationships.

\section{DISCUSSION}

The REA method described by Ståhl et al. (14) and Ståhl and Molin (13) was modified in this study by changing one of the restriction enzymes (Asp 718 to HindIII), shortening the running time of the agarose gels, and utilizing the GelCompar program instead of SIMCA for evaluation. These modifications made the REA easier to handle and allowed us to use a larger proportion of the DNA fragments; because of the long time that elapsed at the original setting, fragments that were smaller than $6.4 \mathrm{~kb}$ ran out of the gels. Thus, in this study almost the entire chromosomal DNA was used for to compare strains.

In order to evaluate whether our results were comparable to the results obtained by Ståhl and Molin (13), a set of L. reuteri strains that previously had been classified by REA (13) was included in the study. In this study, these strains formed a separate cluster at a similarity level of $29 \%$ (cluster 5). The 


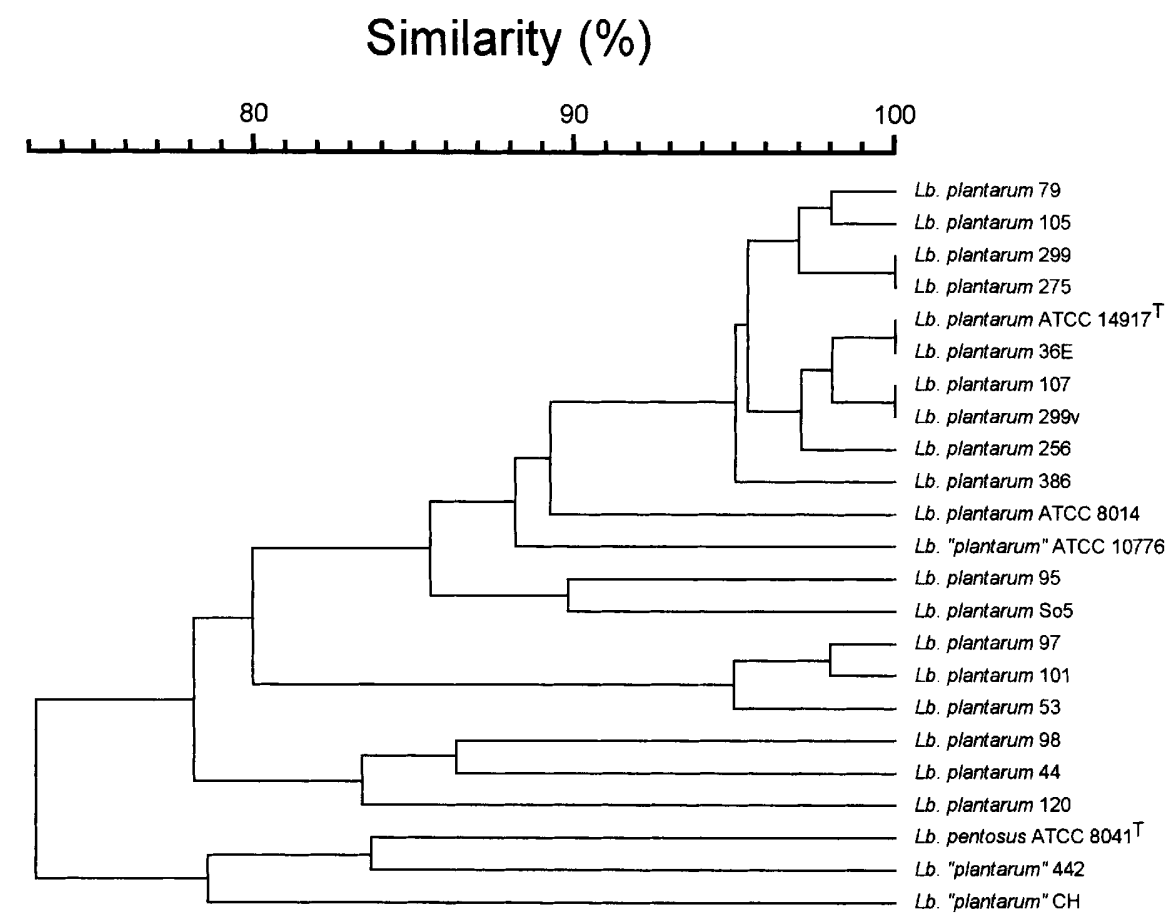

FIG. 5. Dendrogram based on an analysis of combined API $50 \mathrm{CH}$ profiles (obtained at both 30 and $37^{\circ} \mathrm{C}$ ) followed by an analysis in which the Jaccard coefficient and UPGMA were used, showing the phenotypic relationships among the L. plantarum and $L$. pentosus strains included in this study. Lb., Lactobacillus.

formation of subclusters and the relationships among several strains within the subclusters were consistent with the results reported by Ståhl and Molin (13), and this was true despite the fact that one of the restriction enzymes was changed.

All of the L. plantarum strains included in cluster 1 have been shown previously either to exhibit high levels of DNADNA homology with the type strain of $L$. plantarum or to have the same ribopatterns (as determined with EcoRI- and HindIIIcleaved DNA) as $L$. plantarum ATCC $14917^{\mathrm{T}}(6)$. Interestingly, L. plantarum 44 and 120 , both of which merge with cluster 1 at a similarity level of $28 \%$, were found to have different ribopatterns and to exhibit relatively low levels of DNA-DNA homology with the type strain of L. plantarum (6). "L. plantarum" ATCC 10776 was singled out from the rest of the L. plantarum strains by REA (Fig. 1). This is consistent with the DNA-DNA homology data obtained with the type strain of $L$. plantarum $(3,6)$. However, on the basis of the results of phenotypic tests "L. plantarum" ATCC 10776 can be identified as a member of the species (Fig. 5).

Three distinct subclusters were identified within cluster 1 , but the groups did not reflect the sources of isolation. However, we identified some common phenotypic characteristics within subclusters 1a and 1c. Subcluster 1a was made up of three strains that are able to ferment both soluble starch and glycogen, and subcluster 1c (also determined to be a group of closely related strains by PCA) included strains that have the ability to adhere in vitro to human intestinal cell line HT-29 via an $\alpha$-methyl-mannoside-inhibited binding mechanism (15). Two of the strains in subcluster 1c (L. plantarum 299 and 299v) have also been shown to be able to colonize human intestinal mucosa in vivo after oral administration (5). The similarities between strains as well as subclusters of $L$. plantarum were supported by the results obtained by the PCA. We found that phenotypic tests could not be used to group the strains in subcluster 1c (Fig. 5).
REA data can be used to distinguish between very closely related strains. To our knowledge, there are few methods available that are able to identify strains down to such a low level. However, proper gel conditions are necessary i.e., the amount of DNA applied has to be the same, and dots and dirt in the lanes of the gel, as well as normalization errors, must be avoided.

The REA method described in this paper, combined with UPGMA or PCA, has potential for classifying strains at the species level and below. An interesting question is whether this method can be used to estimate levels of relatedness between closely related species. The low levels of similarity between the most distantly related strains belonging to the same species which we observed argue against this possibility. On the other hand, the $L$. pentosus cluster appeared to be closer to the $L$. plantarum clusters than the L. reuteri strains did (Fig. 2). However, to answer this question, the data set has to be expanded, and the REA results have to be compared with results obtained by using available methods for phylogenetic studies.

\section{ACKNOWLEDGMENTS}

We thank Marie Kala for her skillful technical assistance.

This work was supported by the Swedish Agency for Research Cooperation with Developing Countries (SAREC) and the Swedish Work Environment Fund.

\section{REFERENCES}

1. Bauer, W., and J. Vinograd. 1968. The interaction of closed circular DNA with intercalative dyes. I. The superhelix density of SV 40 DNA in the presence and absence of dye. J. Mol. Biol. 33:141-171.

2. Collins, M. D., U. Rodrigues, C. Ash, M. Aguirre, J. A. E. Farrow, A. Martinez-Murcia, B. A. Phillips, A. M. Williams, and S. Wallbanks. 1991. Phylogenetic analysis of the genus Lactobacillus and related lactic acid bacteria as determined by reverse transcriptase sequencing of $16 \mathrm{~S}$ rRNA. FEMS Microbiol. Lett. 77:5-12.

3. Dellaglio, F., V. Bottazi, and M. Vescovo. 1975. Deoxyribonucleic acid homology among Lactobacillus species of the subgenus Streptobacterium Orla Jensen. Int. J. Syst. Bacteriol. 25:160-172. 
4. Efthymiu, C., and P. A. Hansen. 1962. An antigenic analysis of Lactobacillus acidophilus. J. Infect. Dis. 110:258-267.

5. Johansson, M.-L., G. Molin, B. Jeppsson, S. Nobaek, S. Ahrné, and S. Bengmark. 1993. Administration of different Lactobacillus strains in fermented oatmeal soup: in vivo colonization of human intestinal mucosa and effect on the indigenous flora. Appl. Environ. Microbiol. 59:15-20.

6. Johansson, M.-L., G. Molin, B. Pettersson, M. Uhlén, and S. Ahrné. Characterization and species recognition of Lactobacillus plantarum strains by restriction fragment length polymorphism (RFLP) of the 16S rRNA gene. J. Appl. Bacteriol., in press.

7. Johansson, M.-L., M. Quednau, G. Molin, and S. Ahrné. Randomly amplified polymorphic DNA for rapid typing of Lactobacillus plantarum strains. Lett. Appl. Microbiol., in press.

8. Johansson, M.-L., A. I. Sanni, C. Lönner, and G. Molin. 1995. Phenotypically based taxonomy using API $50 \mathrm{CH}$, of lactobacilli from Nigerian ogi, and the occurrence of starch fermenting strains. Int. J. Food Microbiol. 25:159-168.

9. McKay, L. L., and K. A. Baldwin. 1990. Applications for biotechnology: present and future improvements in lactic acid bacteria. FEMS Microbiol. Rev. 87:3-14.

10. Molin, G., B. Jeppsson, M.-L. Johansson, S. Ahrné, S. Nobaek, M. Ståhl, and S. Bengmark. 1993. Numerical taxonomy of Lactobacillus spp. associ- ated with healthy and diseased mucosa of the human intestines. J. Appl. Bacteriol. 74:314-323.

11. Romersburg, H. C. 1984 . Cluster analysis for research. Lifetime Learning Publications, Belmont, Calif.

12. Schleifer, K. H., W. Ludwig, R. Amann, C. Hertel, M. Ehrmann, W. Köhler, and A. Krause. 1992. Phylogenetic relationships of lactic acid bacteria and their identification with nucleic acid probes, p. 23-32. In Les Bactéries Lactiques. Adria Normandie, Centre de Publications de l'Université de Caen, Caen, France.

13. Ståhl, M., and G. Molin. 1994. Classification of Lactobacillus reuteri by restriction endonuclease analysis of chromosomal DNA. Int. J. Syst. Bacteriol. 44:9-14.

14. Ståhl, M., G. Molin, A. Persson, S. Ahrné, and S. Ståhl. 1990. Restriction endonuclease patterns and multivariate analysis as a classification tool for Lactobacillus spp. Int. J. Syst. Bacteriol. 40:189-193.

15. Wold, A. (Department of Clinical Immunology, University of Gothenburg, Gothenburg, Sweden). 1995. Personal communication.

16. Zanoni, P., J. A. E. Farrow, B. A. Phillips, and M. D. Collins. 1987. Lactobacillus pentosus (Fred, Peterson, and Anderson) sp. nov., nom. rev. Int. J. Syst. Bacteriol. 37:339-341. 